\title{
Antimicrobial properties of chitosan extracted from freshwater shrimp (Astacus leptodactylus) caught from Aras Lake
}

\author{
M. Gholampoor ${ }^{1}$, G. Mahmoodi' ${ }^{2}$, A. Moshfegh*3, A. Tehranifard ${ }^{3}$ \\ ${ }^{1}$ Department of Marine Biology, Lahijan Branch, Islamic Azad University, Lahijan, Iran \\ ${ }^{2}$ Department of Biology, Kermanshah Branch, Islamic Azad University, Kermanshah, Iran \\ ${ }^{3}$ Department of Biology, Lahijan Branch, Islamic Azad University, Lahijan, Iran
}

Received: June 2020 Accepted: August 2020

\begin{abstract}
With the emerging of resistant Extracted chitosan showed a stronger microorganisms to conventional antibiotics, as well as consumers' concerns about the side effects of chemical drugs, the tendency for natural bioactive compounds is increasing. Astacus leptodactylus is considered for export and consumption in Iran, but during its processing a large amount of waste material could be produced. The aim of the present study was to investigate the antimicrobial properties of chitosan extracted from $A$. leptodactylus caught from Aras Lake. The shrimp was caught from Lake Aras and transferred to the Microbiology Laboratory of the Islamic Azad University of Lahijan close to ice. Chitosan was extracted from shrimp shells, and methanol extract of shrimp tissue was prepared. Antimicrobial activity of chitosan and extracts were evaluated against Gram-positive bacteria Staphylococcus aureus and Gram-negative bacteria Escherichia coli as well as two species of Aspergillus niger and Candida albicans. *Correspondence A. Moshfegh, Department of Biology, Lahijan Branch, Islamic Azad University, Lahijan, Iran. (Email: moshfeghazam@gmail.com).

inhibitory activity against $S$. aureus compared to $E$. coli. It was also observed that with increasing chitosan concentration, the inhibitory zone diameter increased against both $S$. aureus $(\mathrm{p}=0.001)$ and $E$. coli $(\mathrm{p}=$ 0.122 ). It was also observed that the acidic solution of chitosan had a stronger antibacterial activity than aqueous solution of chitosan. The methanolic extract did not show significant effects on the studied microorganisms. Chitosan had a weak antifungal activity, although it showed a greater effect on $A$. niger than C. albicans.

Keywords: Astacus leptodactylus, Chitosan, Antibacterial activity, Escherichia coli, Staphylococcus aureus

\section{Introduction}

According to significant advances in medical science and access to a variety of drugs, the increasing prevalence of infectious and inflammatory diseases has caused medical researchers to face the problem of pathogen resistance and inefficiency of existing drugs and increase the associated costs (Tanha, Karimzadeh \& Zahmatkesh, 2017). There are
\end{abstract}


concerns about the complications and side effects of common chemical drugs, including the carcinogenic effects of these drugs, which also increase the tendency to replace them with natural origin drugs (Chen et al., 2014). Among these, water resources due to the large area, diversity of biological conditions and consequently the diversity and evolution of existing organisms make it possible to extract a variety of compounds for this purpose (Bhatia \& Chugh, 2015).

Many bioactive compounds have been obtained from marine organisms such as sponges, corals, sea hares, sea sludge, and alga. Also, wastes of production and processing plants of fish cause environmental problems and increase the costs of maintenance and disposal of these wastes. Surely, high value-added compounds and products can be extracted from these wastes if they were properly processed. (Kumar \& Pal, 2016).

A. leptodactylus is widely consumed in many countries as a commercial and special aquatic food due to its high content of omega3 fatty acids such as Eicosapentaenoic acid and Docosahexaenoic acid (Martin, Carter \& Davis, 2000; Harlioğlu, 2004). A. leptodactylus is the only genus of Astacus habitats in Iran caught from internal freshwater sources. Among the water resources, the reservoir lake behind Aras Dam located in Poldasht city of West Azerbaijan province is one of the main habitats and the only commercial fishing place of this species in Iran, which more than 200 tons of this species is exported annually to European countries (Karimpour, Harlioğlu
\& Aksu, 2011). During the processes of the Astacus leptodactylus, about $15 \%$ of the harvesting is converted to fillet and the remaining will be as waste (Moody \& Martin, 2000).

Extraction of beneficial compounds from these wastes and using them in animal diets as an additive or supplement has been reported in previous studies (Kumar \& Pal, 2016; Flick, 1994). It has also been reported that king shrimp's shell has significant amounts of Astaxanthin pigment, flavoring compounds, chitin, etc. (Escobar, Wells \& Waikar, 1991; Meyers \& Lee, 1989; Kumar \& Pal, 2016). Chitin is a Mucopolysaccharid and has a structural role, which is found in abundance in the external skeleton of arthropods such as shrimp, crab and in insects as well as Cryptogamae. Some applications of chitin in fish rearing and medical sciences include disinfection, increase of seed growth, protective coating of seeds against pests, antifungus, treatment of skin burns, orthopedics, enrichment with a variety of drugs, antibiotics and vitamins. Also, one of the most important derivatives of chitin is a compound called chitosan, which unlike chemical polymer compounds, is non-toxic and biodegradable in nature. Chitosan is used in a wide range of industries including pharmaceuticals, agriculture, food industries, water treatment, biotechnology, medicine, cosmetics, textiles, papermaking, etc. Chitosan can be used in the food industry as antibacterial, antifungal, antioxidant, food coatings, food concentrates, strengthening, water absorption, transparency in products such as juices, to prevent food color change, 
gel production and reduce fat absorption in fried products (Kumar, 2000).

Fortunately, in the southern parts of the country, there are good reserves of shrimp and crabs for chitin, but unfortunately, their skin is removed and discarded without use after fishing. Due to the scarcity and dispersion of aquaculture processing plants, the total amount of catch is divided and scattered in different areas after reaching the shore, therefore there is no clear plan for the optimal use of these wastes in the aquaculture sector. Also, high perishability, seasonal fishing of these fish and high temperature of fishing areas are other important reasons which make it impossible for the productivity of these wastes in the South. Therefore, due to the existence of vast water resources in the North and South of the country and also the significant reserves of crustaceans in these waters, the necessary measures have been taken in terms of productivity of these vast resources and the necessary planning to extract these valuable compounds, also taking into account the high potential of the country, while meeting domestic needs and eliminating the need to import this product, it provided the necessary grounds for its export to other parts of the world and provided currency for the country.

Therefore, the aim of the present study was to extract Chitosan from A. leptodactylus that was harvested from Lake Aras and to investigate its antimicrobial properties.

\section{Materials and Methods}

\section{Equipment and Ingredients}

The materials, culture media and instruments, which were used in this study, were listed in Table 1.

Table 1. Applied equipment and used ingredients

\begin{tabular}{lll}
\hline Row & Apparatus and material & brand \\
\cline { 2 - 3 } 1 & Autoclave & Hyrayama,Japan \\
2 & Bain-marie & Memmert,Germany \\
3 & Incubator & Memmet,Germany \\
4 & microbial hood & Behdad,Iran \\
5 & Centrifuge & Pars Azma C, Iran \\
6 & Oven & Memmert,Germany \\
7 & DMSO & QueLAB \\
8 & Muller Hinton Agar growth medium (MHA) & Merck \\
9 & Muller Hinton Broth growth medium & Merck \\
10 & Tryptic Soy Broth growth medium & Merck \\
11 & Antibiotic disc & Padtan TEB \\
\hline
\end{tabular}




\section{Providing samples}

To conduct the research in summer, $A$. leptodactylus were caught from Aras Dam Lake located in West Azerbaijan Province and transferred to the Microbiology Laboratory of Islamic Azad University, Lahijan Branch for identification and preparation of extract processes. $A$. leptodactylus samples were identified by using the available identification keys.

\section{Extraction of chitosan}

After washing, the waste and shell of $A$. leptodactylus were manually removed from the flesh and transferred to an oven at $37{ }^{\circ} \mathrm{C}$ and allowed to dry for 24 hours. The waste and shell were then ground and stored separately in a dark glass in a freezer at -20 ${ }^{\circ} \mathrm{C}$ until use. $2 \mathrm{M}$ profit was added to $5 \mathrm{~g}$ of dried A. leptodactylus shell powder, and it was incubated at $25^{\circ} \mathrm{C}$ for 15 minutes to deproteinize the sample. Then the supernatant was centrifuged at $2000 \mathrm{rpm}$ for 10 minutes. The surface alkaline liquid was separated and discarded. The resulting precipitate was washed several times with distilled water. 25 $\mathrm{ml}$ of $0.5 \mathrm{M}$ sulfuric acid was added to the precipitate and placed at $50^{\circ} \mathrm{C}$ for 16 hours to remove minerals. The sample was then centrifuged at $2000 \mathrm{rpm}$ for 10 minutes. Then the precipitate was washed with distilled water several times. In order to purify the extracted chitin, dried A. leptodactylus with $2 \%$ sodium hydroxide solution (1: 30$)$ was deproteinized for 2 hours at $90^{\circ} \mathrm{C}$. Then the resulting precipitate and the alkaline mixture were washed. To extract chitosan from the above precipitate mixtures, it was placed in the presence of $10 \%$ acetic acid at a ratio of 10: $1(\mathrm{w} / \mathrm{v})$ at $60^{\circ} \mathrm{C}$ for 6 hours and then the crude and pure chitin were separated by centrifugation for 15 minutes at $4000 \mathrm{rpm}$.
The PH of the superior phase, which contains chitosan in acetic acid solution, was reduced to about 9 with Sodium 4 molar. At this point, the chitosan was suspended in solution and then precipitated. Both resulting residues, crude chitin and chitosan, were washed with distilled water, ethanol and acetone, and were dried at $20{ }^{\circ} \mathrm{C}$. The resulting precipitate was placed in an oven at $105^{\circ} \mathrm{C}$ for 24 hours and the dry sample weight was determined (Chang \& Tsai, 1997).

\section{Preparation of methanolic extract}

Each muscle tissue was cut into small pieces. This tissue was poured into a sterile container containing $96 \%$ methanol in a ratio of $1 \mathrm{ml}$ distilled water with $4 \mathrm{~g}$ of tissue and placed in laboratory temperature for one week, after which the solution was passed through filter paper. About $200 \mathrm{ml}$ of crude methanolic extract was evaporated at room temperature in a rotary apparatus under vacuum. This methanolic extract was then stored at $-20{ }^{\circ} \mathrm{C}$. Another $200 \mathrm{ml}$ of crude methanolic extract with $200 \mathrm{ml}$ of chloroform was poured into another container, which formed two separate phases after a few minutes. After 3 days, the chloroform section was separated and evaporated. The obtained chloroform extract was stored at $-20{ }^{\circ} \mathrm{C}$. In order to use chitosan extracts obtained during the mentioned processes, for microbial tests and sensitivity determination, was directly used in aqueous and acidic solvents (1 M) and along with DMSO applied for anti-fungal tests). According to the sources, it is better to mix the extracted chitosan with $45 \%$ sodium hydroxide solution in a ratio of $1: 10$. The above samples were transferred to a bainmarie at $85^{\circ} \mathrm{C}$ at intervals of 20 and 45 minutes. 
The studied microorganisms in the present study, the bacterial strains of $S$. aureus (PTCC: 1113) and E. coli (PTCC: 1763) as well as the fungal and yeast strains of Aspergillus niger (PTCC: 5154) and Candida albicans (PTCC: 5027) were collected and prepared as lyophilized from the Iranian Bacterial Collection Center, Iran was prepared as lyophilized and were used to study the antibacterial effects of chitosan extracted from freshwater king prawns. Activation of bacterial strains was performed by transferring the contents of each vial in screw tubes containing $10 \mathrm{ml}$ of Tryptic Soy Broth and incubating for 24 hours at $37^{\circ} \mathrm{C}$. The fungi were also activated in Potato Dextrose Agar culture medium and stored for 24 hours at $30{ }^{\circ} \mathrm{C}$. To prepare the bacterial suspension equivalent to $0.5 \mathrm{McFarland}$ in tubes containing Mueller Hinton broth medium, 4 to 5 colonies were removed from the culture medium after 18 to 24 hours of inoculation and inoculated into TSB medium. The tube was shaken vertically to compare the resulting uniform solution with the semiMcFarland turbidity. The light absorption of the 0.5 McFarland suspension at $625 \mathrm{~nm}$ should have an absorption of 0.8-0.13. Finally, if the above conditions were met, the microbial suspension would have a concentration equivalent to $1-2 \times 10^{8}$ $\mathrm{CFU} / \mathrm{ml}$.

\section{Disk diffusion method}

First, after 15 minutes of preparing 0.5 McFarland turbidity from the desired strains, culturing was uniformly carried out by a sterile swap on MHA medium and then sterile blank disks were inoculated with $20 \mu \mathrm{l}$ of specific doses of chitosan extract. Extraction of A. leptodactylus was transferred by sterile forceps to the cultured media. Finally, the plates were incubated at $37{ }^{\circ} \mathrm{C}$ for 18 hours and after the incubation time the plates were examined for observation or non-observation of the growth halo diameter and the desired halos were measured.

\section{Well diffusion well}

Similar to the disk diffusion method, the bacteria were first cultured uniformly and then pasteurized using a sterile pipette, $6 \mathrm{~mm}$ wells with a capacity of $100 \mu$ were punched in agar and cooled molten agar was used to block wells in order to prevent extracts from escaping from the bottom of each well. Finally, chitosan extracts extracted from $A$. leptodactylus was transferred by means of a sample from specific dilutions into each well embedded in agar. The plates were incubated at $37^{\circ} \mathrm{C}$ for 18 hours and after the incubation period the plates were examined for observation or non-observation of the growth halo diameter.

\section{Determination of MIC and MBC}

Macro Dilution Broth method was used to determine MIC and MBC. MIC is actually the minimum concentration of antimicrobial agent that can inhibit the growth of microorganisms, and if this agent is removed from the environment, the microorganism will be able to grow again. $\mathrm{MBC}$ is the minimum concentration of antimicrobial agent that can kill $99.9 \%$ of bacteria and if secondary culture is carried out, less than $0.1 \%$ of primary bacteria can grow. In this method, different dilutions of chitosan extract were prepared along with Mueller Hinton broth culture area and each of the bacteria were added and inoculated with a precise sampler in each of the tubes 
containing the culture medium. Each bacterium equivalent to $0.5 \mathrm{McF}$ arland in the amount of $300 \mu \mathrm{l}$ along with dilution of 10 , 20, 40,60, 80, $100 \mathrm{mg} / \mathrm{ml}$ of chitosan extract was inoculated to Mueller Hinton broth culture medium and incubated for $24 \mathrm{~h}$ at 37 ${ }^{\circ} \mathrm{C}$. For each series of diluted forms, a control + (including bacteria + culture medium), control - (including extracted chitosan + culture medium) and a blank tube were considered to control the culture medium in each series. After incubation time, each tube was evaluated for turbidity or non-turbidity in which no growth was observed it considered as MIC. In order to determine the MBC from each concentration, surface culture was performed in a plate containing Nutrient Agar incubated for 24 hours at 37 ${ }^{\circ} \mathrm{C}$.

\section{Antifungal Test Method}

The selected fungi for testing were Candida albicans and Aspergillus niger which were collected from the Iranian Collection Center of Bacteria and Fungi and used for antifungal test. In order to evaluate the antifungal effect of extracts Nutrient broth at a temperature of 35 degrees was used and compared to itraconazole as a reference drug.

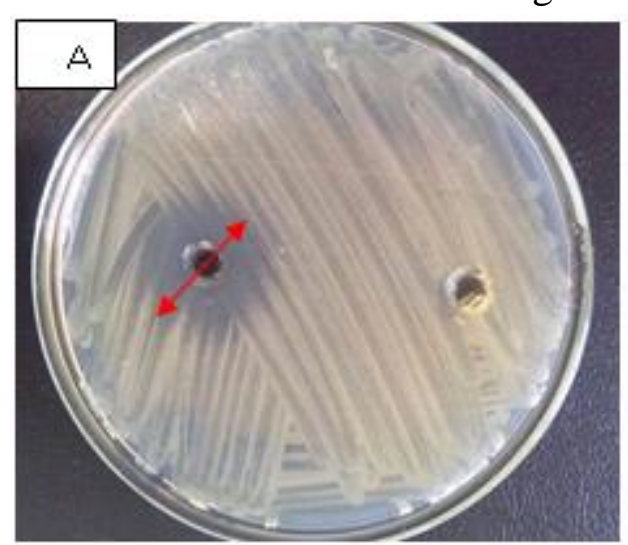

Concentrations of 80 and $100 \mathrm{mg} / \mathrm{ml}$ of extracts were dissolved in DMSO solvent and added in tubes of $0.25 \mathrm{McF}$ arland Nutrient broth along with anti-fungal suspension. It was then incubated at $35^{\circ} \mathrm{C}$. The growth of the fungi was examined for a week and the MIC was determined.

\section{Results}

\section{Antibiogram Test}

The results related to the antimicrobial effect of chitosan in terms of growth inhibition zone (mm) against $S$. aureus and E. coli is shown in Table 2 and Figure 1. The extracted chitosan showed the maximum inhibitory activity against Gram-positive bacteria. $S$. aureus showed that at all concentrations studied the diameter of the inhibitory aura against $S$. aureus was more than $E$. coli bacteria. It was also observed that with increasing chitosan concentration, antibacterial effects against both $S$. aureus (p $=0.001)$ and $E$. coli $(\mathrm{p}=0.012)$ were significant and with increasing chitosan concentration, the diameter of the inhibition halo was significantly higher. In addition, no significant difference was observed between the results of the well and disk method ( $p$ $<0.05)$.

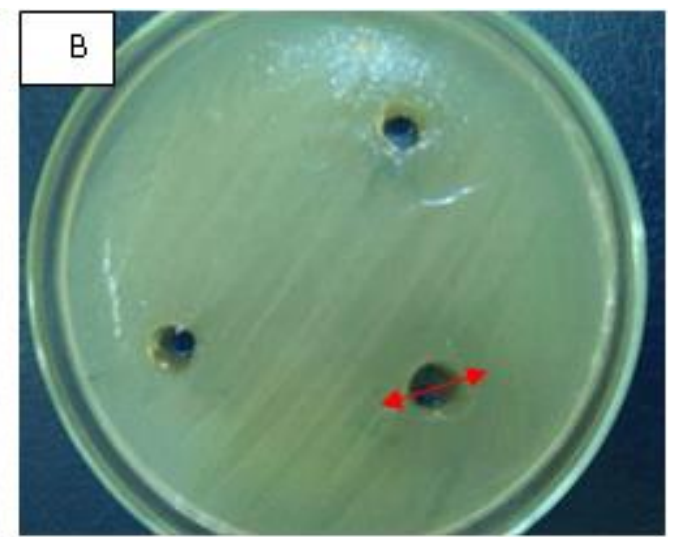

Figure 1. Effect of extracted chitosan on S. aureus (A) and E. coli (B) 
The results of the minimum inhibitory concentration (MIC) and maximum lethal concentration (MBC) of chitosan extracted against the studied bacteria are shown in Table 3. The lowest amount of MIC and MBC chitosan extracted against $S$. aureus was observed while no significant effects were observed against E. coli. It was also observed that the acidic solution of chitosan had stronger antibacterial activity than the aqueous solution of chitosan, so that the MIC and MBC of the acidic solution of chitosan against $S$. aureus were 20 and $40 \mathrm{mg} / \mathrm{ml}$, respectively, and these values were equal for the aqueous solution of chitosan extract, 40 and $80 \mathrm{mg} / \mathrm{ml}$, respectively. No inhibitory and inactivating effects were observed in $E$. coli.

Table 2. Antimicrobial effect of chitosan in different concentrations in terms of growth inhibition zone $(\mathrm{mm})$

\begin{tabular}{lllllll}
\hline & $\mathbf{2 0}$ & $\mathbf{4 0}$ & $\mathbf{6 0}$ & $\mathbf{8 0}$ & $\mathbf{1 0 0}$ & P value \\
\cline { 2 - 7 } & & & & & & \\
\hline S. aureus & 4 & 11 & 15 & 17 & 19 & 0.001 \\
PTCC 1113 & & 0 & 9 & 13 & 14 & 0.012 \\
\hline $\begin{array}{l}\text { E. coli } \\
\text { PTCC 1763 }\end{array}$ & 0 & & & & & \\
\hline
\end{tabular}

Table 3. Effects of minimum inhibitory concentration (MIC) and minimum lethal concentration (MBC) of acidic and aqueous solution of chitosan

\begin{tabular}{|c|c|c|c|c|}
\hline & \multicolumn{2}{|c|}{$\begin{array}{l}\text { Aqueous solution of chitosan extracted } \\
\text { from } A \text {. leptodactylus }\end{array}$} & \multicolumn{2}{|c|}{$\begin{array}{l}\text { Acidic solution of chitosan } \\
\text { extracted from } A \text {. leptodactylus }\end{array}$} \\
\hline & $\mathrm{MBC} \mathrm{mg} / \mathrm{ml}$ & $\mathrm{MIC} \mathrm{mg} / \mathrm{ml}$ & $\mathrm{MBC} \mathrm{mg} / \mathrm{ml}$ & $\mathrm{MIC} \mathrm{mg} / \mathrm{ml}$ \\
\hline S. aureus & 80 & 40 & 40 & 20 \\
\hline \multicolumn{5}{|l|}{ PTCC1113 } \\
\hline E.coli PTCC 1763 & No effect & No effect & No effect & No effect \\
\hline
\end{tabular}

The effect of methanolic extract of $A$. leptodactylus against the studied microorganisms is shown in Figure 2. In the studies obtained from the effect of total extract to the determination of susceptibility to disk and welling methods, except for total concentration by disk method, which had a growth inhibition zone with a diameter of $9 \mathrm{~mm}$ against $S$. aureus, no significant effects of methanolic extract of A. leptodactylus were observed against the studied microorganisms. 

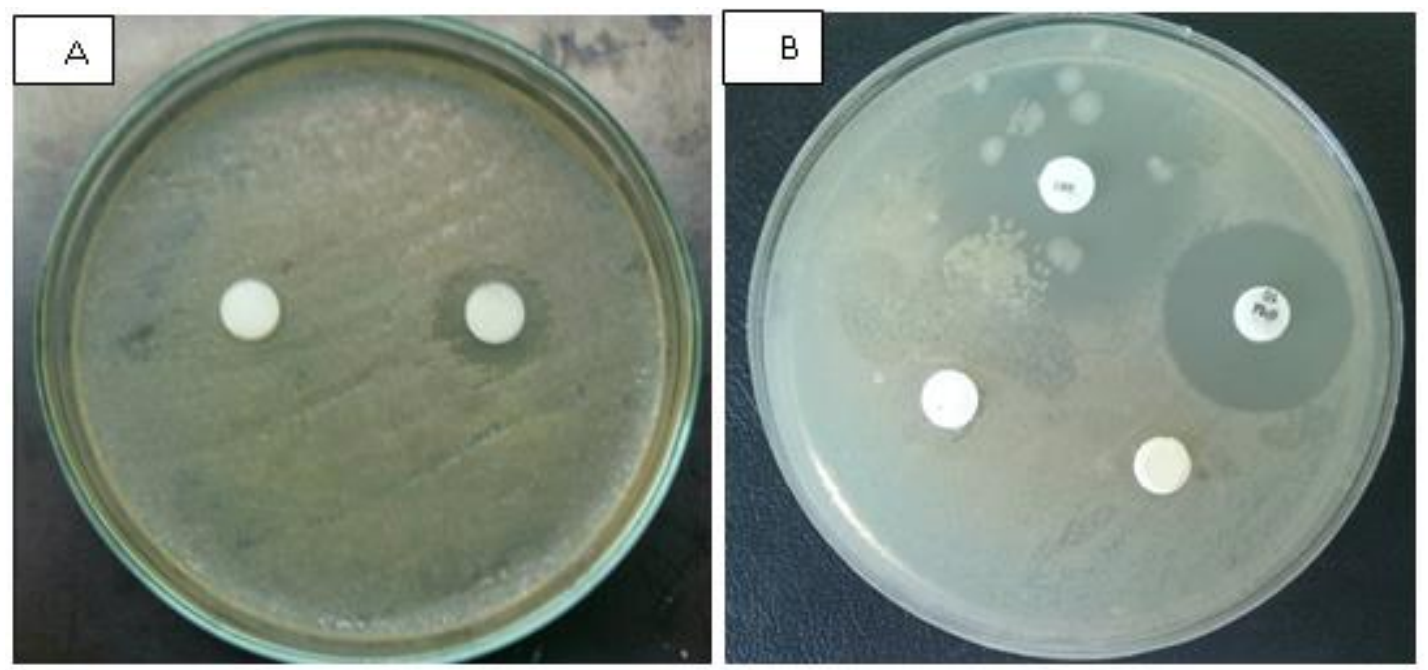

Figure 2. Effect of disc containing alcoholic extract of A. leptodactylus against S. aureus (A) and E. coli (B)

Assaying the antifungal activity of chitosan extracted against $C$. albicans and A. niger showed that acid-soluble chitosan (extracted from acid as a chitosan solvent and used in powder form) had a greater effect on A. niger, although it did not show significant inhibition and did not prevent hexadecimal growth, but to some extent an aura of growth was observed (Figure 3).

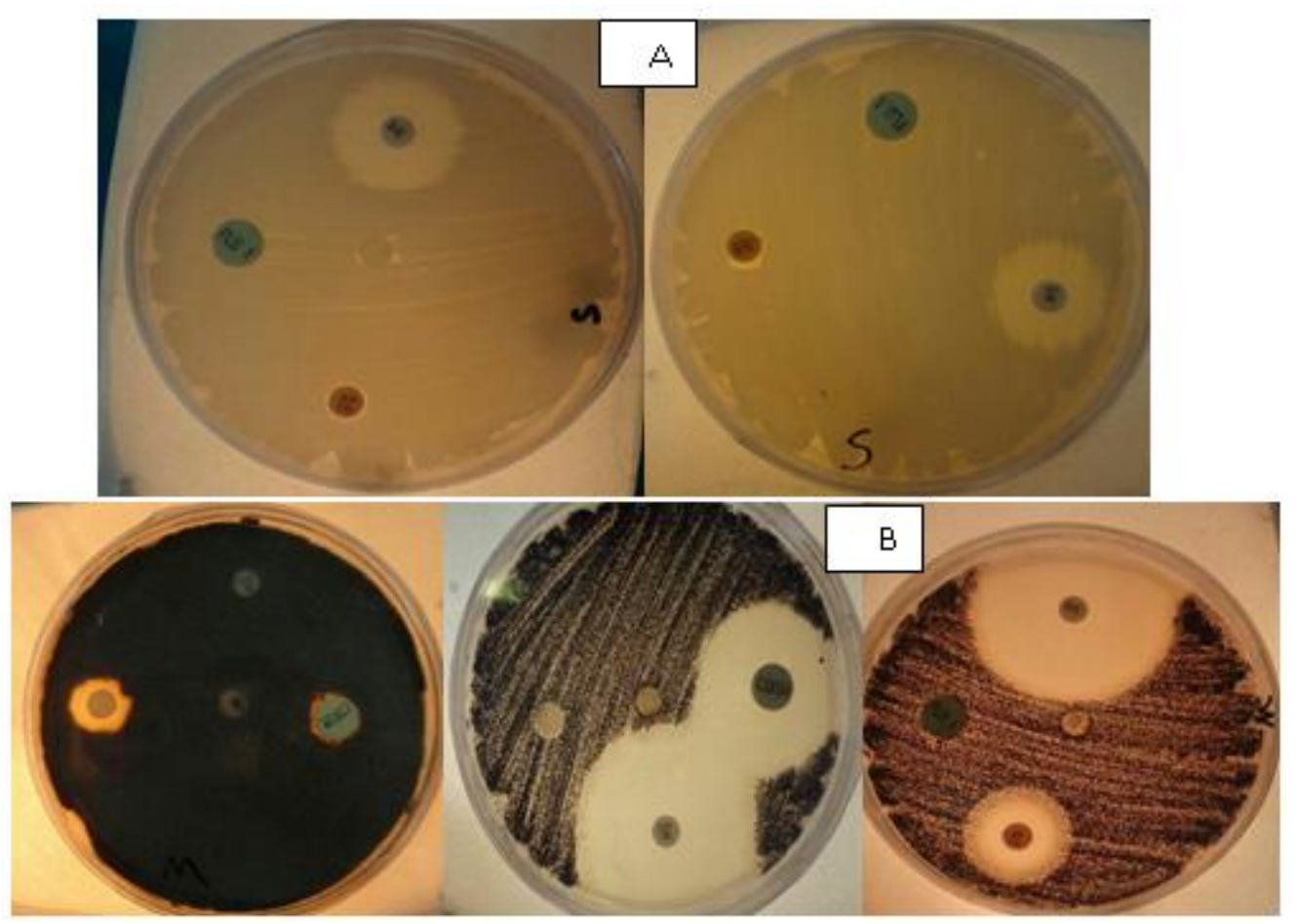

Figure 3. Antibiogram tests on Aspergillus niger (A) and Candida albicans (B). 
As shown in Figure 4, the diameter of the nongrowth halos of each bacterium relative to the extracted acidic and aqueous extracts compared to standard antibiotics shows that in general $S$. aureus was more sensitive to the studied antibiotics compared to $E$. coli.

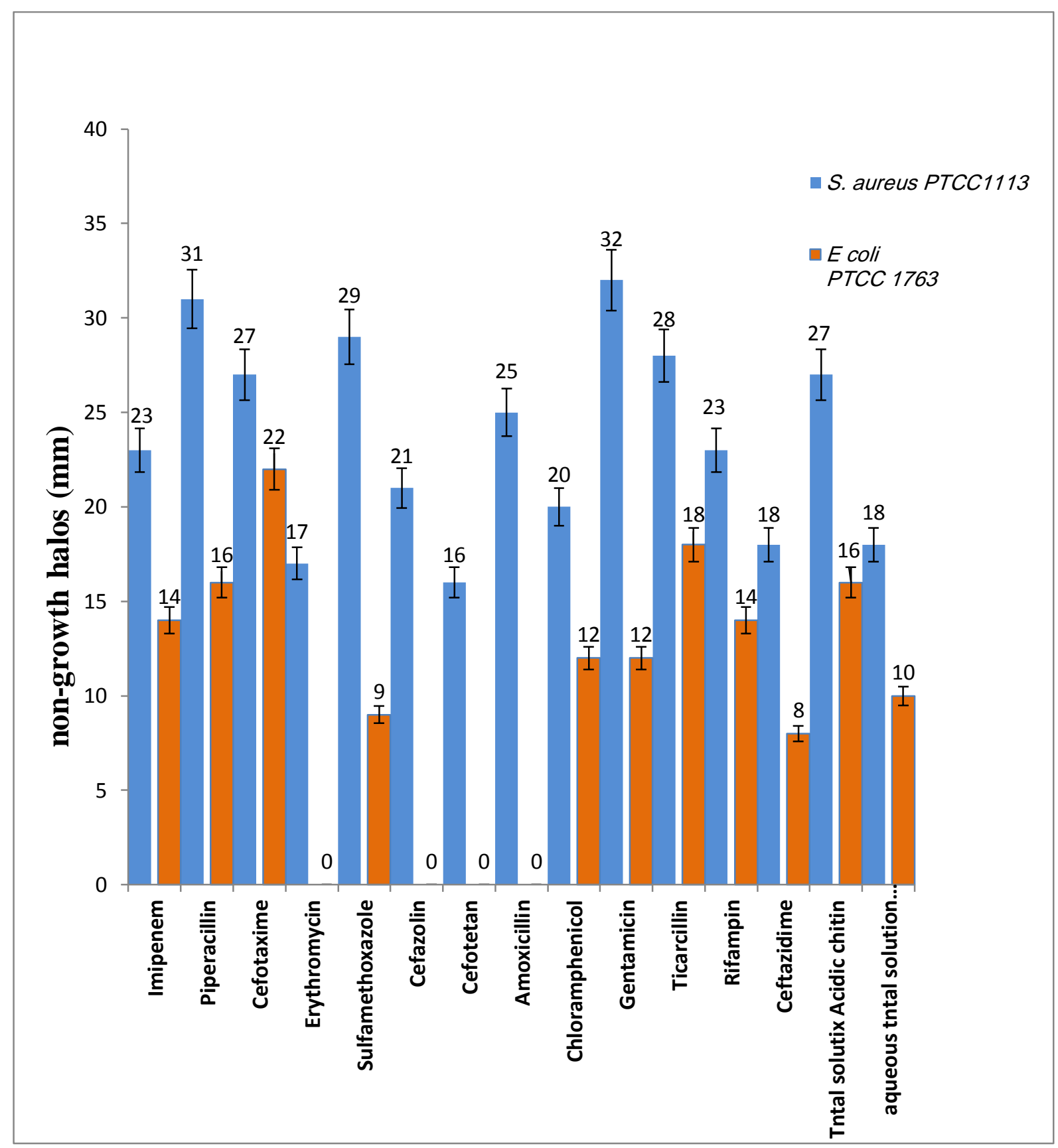

Figure 4. Comparison of growth inhibition zone of antibiotics and total acidic and aqueous extracts on standard strains of $E$ coli and S. aureus 


\section{Discussion}

Due to the concerns caused by the side effects of common chemical drugs, including the carcinogenic effects of these drugs and the emergence of antibiotic resistance of microorganisms, the tendency to use compounds of natural origin is increasing day by day (Chen, 2014). Chitosan was extracted from the leachate of A. leptodactylus and its antibacterial activity was evaluated. The results show a stronger effect of chitosan against $S$. aureus bacteria compared to $E$. coli. Also, the antifungal activity of the studied chitosan was weaker than its antibacterial activity.

In most previous studies, it has been reported that chitosan generally has a stronger bactericidal effect on gram-positive than gram-negative bacteria (Rout, 2001; Másson et al., 2008). These results are consistent with the results of the present study, which showed that chitosan has the greatest effect of growth inhibition on $S$. aureus compared to E. coli. On the other hand, some studies have reported higher antimicrobial activity against gram-negative bacteria (Tareq et al., 2013; Chen, Liau \& Tsai, 1998; Chung et al., 2004) and some have not reported significant differences in this regard (Chen et al., 1996). Therefore, more and more comprehensive studies are necessary. In the study of Taheri et al. (2013) it was observed that chitosan had a stronger antibacterial effect against $S$. aureus compared to E. coli, although in the study of these researchers most effects were observed against Vibrio ogava (Taheri, Seyfan \& Jalalinezhad, 2013). Results of the study of Taheri et al. (2013) on the stronger antibacterial effects of chitosan against $S$. aureus compared with E. coli, is consistent with the results of the present study. It has been reported that one of the main reasons for higher antimicrobial activities of chitosan against gram-positive compared to gramnegative bacteria is the difference between the cell surfaces as a protective membrane around the cell wall (Isamu et al. 2003). Other reasons for the differences in antimicrobial activity of chitin and chitosan are relevant to different sources include a) type and age of pathogenic microorganisms, b) physicochemical properties of chitin and chitosan, c) source of chitin and chitosan and d) Extraction conditions (Hongpattarakere \& Riyaphan, 2008).

In addition, the relatively weak antifungal activity of chitin and chitosan was attributed to the effect of antimicrobial compounds on the solid cell wall of fungi, including chitin and glucan. Can be confirmed antibacterial activity (Másson, Holappa \& Hjálmarsdóttir, 2008). On the other hand, chitosan showed significant antifungal properties (Burrows, 2007).

In the present study, with increasing chitosan concentration, the inhibitory activity against the studied microorganisms increased, which is consistent with the results of the previous studies. Taheri et al. (2014) reported that among the concentrations of 5, 7 and $10 \%$ chitosan, the highest inhibitory activity was observed for $10 \%$ solution (Taheri, Seyfan \& Jalalinezhad, 2013). These results indicate the fact that chitosan has a certain antimicrobial effect, so increasing the concentration of chitosan also increases its effectiveness and lethality. However, some researchers have reported that chitosan's 
antibacterial activity does not increase by increasing its concentration, which may be due to differences in test conditions. Also, increased chitosan viscosity at higher concentrations and limited number of effective chitosan contacts with the cell surface of bacterial cells are other reasons for this phenomenon.

In this study, the effectiveness of the extracted chitosan, which was dissolved in acid, was more potent than the aqueous solution, which can be attributed to both the type of acidic solution and the effect of the gram-positive wall. Taheri et al. (2013) reported that acetic acid soluble chitosan showed stronger antibacterial activity compared to water soluble chitosan, which is consistent with the results of Taheri et al. (2013) study. Chitosan is in pH less than 6 polycationic and reacts easily with negatively charged compounds such as proteins, anionic polysaccharides, fatty acids, and phospholipids, destroying bacterial membranes. In fact, the antibacterial properties of chitosan depend on the protonation of amino groups, which can react with a negative charge on the cell surface and cause bacterial cell destruction. It has also been reported that because chitosan is insoluble at $\mathrm{pH}=7$ and has no amino groups, it has low antimicrobial activity (Taheri et al., 2013).

Also, in the present study, it was observed that the extracted chitosan showed acceptable inhibitory activity in comparison with the studied antibiotics, to which some bacteria had antibiotic resistance, and these results are consistent with the results of Taheri et al. (2013).
Chitin and chitosan have a wide range of microbial activities against gram-positive bacteria, gram-negative bacteria and fungi, although the amount of this activity is different for each category. This antimicrobial activity, which in fact reflects the defense barrier against microorganisms with chitinous shells, provides significant potential for use in a variety of drugs. Although the mechanism of antimicrobial activity of chitin and chitosan is not well known, the acceptable mechanism for this activity is the interaction between the positive charges of the chitin and chitosan molecules and the negative charges of the cell membrane surface of pathogens. In this regard, more positive charges of chitosan surface and more antimicrobial activity can be expected through this mechanism. However, further studies are needed to be better understood the mechanism of antimicrobial activity of chitin and chitosan.

Overall, the significant effect of extracted chitosan extracts, especially against grampositive $S$. aureus bacteria has been observed, and can be suggested that the waste of marine organisms such as shrimp skin be used as an available and inexpensive source for the production of antibacterial and inhibitory drugs. One of the limitations of the present study was the lack of chitosan production to measure its degree of distillation.

\section{References}

Bhatia, P., \& Chugh, A., 2015. Role of marine bioprospecting contracts in developing access and benefit sharing mechanism for marine traditional knowledge holders in the pharmaceutical industry. 
Global Ecology and Conservation, 3, 176187.

Burrows, F., Louime, C., Abazinge, M., \& Onokpise, O., 2007. Extraction and evaluation of chitosan from crab exoskeleton as a seed fungicide and plant growth enhancer. American-Eurasian Journal of Agricultural and Environmental Science, 2(2), 103-111.

Chang, K. L. B., \& Tsai, G., 1997. Response surface optimization and kinetics of isolating chitin from pink shrimp (Solenocera melantho) shell waste. Journal of agricultural and food chemistry, 45(5), 19001904.

Chen, C. S., Liau, W. Y., \& Tsai, G. J., 1998. Antibacterial effects of N-sulfonated and Nsulfobenzoyl chitosan and application to oyster preservation. Journal of Food Protection, 61(9), 1124-1128.

Chen, J., Wang, F., Liu, Q., \& Du, J., 2014. Antibacterial polymeric nanostructures for biomedical applications. Chemical communications, 50(93), 14482-14493.

CHEN, M. C., YEH, G. H. C., \& CHIANG, B. H., 1996. Antimicrobial and physicochemical properties of methylcellulose and chitosan films containing a preservative. Journal of Food Processing and Preservation, 20(5), 379390.

Chung, Y. C., Su, Y. P., Chen, C. C., Jia, G., Wang, H. L., Wu, J. G., \& Lin, J. G., 2004. Relationship between antibacterial activity of chitosan and surface characteristics of cell wall. Acta pharmacologica sinica, 25(7), 932-936.

Escobar, F. A., Wells, J. H., \& Waikar, A. M. 1991. Simulation based performance analysis of crawfish processing operations. Journal of food process engineering, 14(2), 147-162.

Flick, G. J., Lovell, R. T., ENRIQUEZIBARRA, L. G., \& Arganosa, G. C., 1994. Changes in nitrogenous compounds in freshwater crayfish (Procambarus clarkii) tail meat stored in ice. Journal of muscle foods, 5(2), 105-118.

Harlioğlu, M. M., 2004. The present situation of freshwater crayfish, Astacus leptodactylus (Eschscholtz, 1823) in Turkey. Aquaculture, 230(1-4), 181-187.

Hongpattarakere, T., \& Riyaphan, O. 2008. Effect of deacetylation conditions on antimicrobial activity of chitosans prepared from carapace of black tiger shrimp. Songklanakarin Journal of Science \& Technology, 30.

Karimpour, M., Harlioğlu, M. M., \& Aksu, Ö., 2011. Status of freshwater crayfish (Astacus leptodactylus) in Iran. Knowledge and Management of Aquatic Ecosystems, (401), 18.

Kumar, M. N. R., 2000. A review of chitin and chitosan applications. Reactive and functional polymers, 46(1), 1-27.

Kumar, M. S., \& Pal, A. K., 2016. A review of bioactive compounds from marine organisms with special mention on the potential of marine sponges in pharmacological applications. Journal of the Marine Biological Association of India, 58(1), 84 .

Martin, R. E., Carter, E. P., \& Davis, L. M. (Eds.). 2000. Marine and freshwater products handbook. CRC Press.

Másson, M., Holappa, J., Hjálmarsdóttir, M. OV R únarsson, T. Nevalainen, \& T. Järvinen 2008. "Antimicrobial activity of piperazine 
derivatives of chitosan,". Carbohydrate polymers, 74(3), 566-571.

Másson, M., Holappa, J., Hjálmarsdóttir, M., Rúnarsson, Ö. V., Nevalainen, T., \& Järvinen, T., 2008. Antimicrobial activity of piperazine derivatives of chitosan. Carbohydrate polymers, 74(3), 566-571.

Moody, M.W., 2000. Martin R. Handling and processing crawfish. Marine and freshwater products handbook Lancaster, PA: Technomic Publishing Co, 309-22.

No, H. K., Meyers, S. P., \& Lee, K. S., 1989. Isolation and characterization of chitin from crawfish shell waste. Journal of Agricultural and Food Chemistry, 37(3), 575-579.

Rout, S. K., 2001. Physicochemical, Functional and Spectroscopic Analysis of Crawfish Chitin and Chitosan as Affected by Process Modification.
Taheri, A., Seyfan, A., \& Jalalinezhad, S., 2013. Antimicrobial and antifungal effects of acid and water-soluble chitosan extracted from Indian shrimp (Fenneropenaeus indicus) shell. Journal of Fasa University of Medical Sciences, 3(1), 49-55.

Tanha, N., Karimzadeh, K., \& Zahmatkesh, A., 2017. A study on the antimicrobial activities of chitin and chitosan extracted from freshwater prawn shells (Macrobrachium nipponense). International Journal of Health Studies, 3(3).

Tareq, A., Alam, M., Raza, S., Sarwar, T., Fardous, Z., Chowdhury, A. Z., \& Hossain, S., 2013. Comparative study of antibacterial activity of chitin and chemically treated chitosan prepared from shrimp (Macrobrachium Rosenbergii) shell waste. Journal of Virology and Microbiology, 9. 